\title{
Kinetic Retraction at the Onset of Concomitant Crystallization
}

\author{
Tonglei Li \\ Purdue University, West Lafayette, United States of America; \\ tonglei@purdue.edu
}

We have recently studied a diarylamine compound, tolfenamic acid (TFA), and examined its solution chemistry, crystallization kinetics, and molecular interactions. The polymorphic system typically crystalizes as From I or Form II, or both concurrently, with Form I being the most stable at room temperature. Both polymorphs are composed of hydrogen-bonded, carboxyl homodimers as the supramolecular synthon in their respective crystal structures. One interesting kinetic phenomenon that we experimentally discovered was an intermediate or transitional retraction of the mass composition of Form I in crystallized samples over the course of concomitant crystallization. The composition retraction bears two characteristic attributes, the retraction depth and the onset fraction. The former quantifies the maximal extent to which the Form I composition retracts prior to elevation, whereas the later attribute characterizes the initially measured Form I composition. Conversely, during solvent-mediated phase transformation, the mass composition of Form I monotonically increases and only Form II nucleates initially. We further learned through population balance simulations that this characteristically kinetic phenomenon is a sufficient condition indictor of concomitant crystallization of polymorphic systems. Interestingly, when experimental observation is made at a later time after the kinetic retraction, it seems unlikely to kinetically differentiate the two crystallization pathways.

Keywords: concomitant polymorphism; solvent-mediated phase transition; population balance modeling 